\title{
Alpha-2-macroglobulin and hyaluronic acid as fibromarkers in patients with chronic hepatitis $\mathrm{C}$
}

\author{
Pitekova $\mathrm{B}^{1}$, Kupcova $\mathrm{V}^{2}$, Uhlikova $\mathrm{E}^{1}$, Mojto $\mathrm{V}^{2}$, Turecky $\mathrm{L}^{1}$ \\ Institute of Medical Chemistry, Biochemistry and Clinical Biochemistry, Medical School, \\ Comenius University, Bratislava, Slovakia. ladislav.turecky@fmed.uniba.sk
}

\begin{abstract}
BACKGROUND: Liver fibrosis is the final common pathway of chronic liver diseases of various etiology. From the practical standpoint, it would be ideal to have a noninvasive fibromarker. The aim of our study was to investigate the levels of alpha-2-macroglobulin, potential fibromarker, in correlation to histological staging and another potential fibromarker, hyaluronic acid, in patients with chronic hepatitis $\mathrm{C}$.

METHODS: Population groups in this study consisted of 51 healthy volunteers and 54 patients with chronic hepatitis C. Liver biopsies were obtained under ultrasound guidance. Alpha-2-macroglobulin was determined by electroimmunodiffusion and hyaluronic acid with enzyme-linked binding protein assay.

RESULTS: Both potential fibromarkers, alpha-2-macroglobulin and hyaluronic acid, were increased in patients with chronic hepatitis $\mathrm{C}$. The alpha-2-macroglobulin levels were not significantly increased in the groups F0F1. In the groups F2-F4, alpha-2-macroglobulin levels were significantly higher than in the control group. The changes of hyaluronic acid were similar to changes of alpha-2-macroglobulin. Regression analysis showed a significant correlation between hyaluronic acid and alpha-2-macroglobulin levels.

CONCLUSIONS: According to the results of our study, it can be concluded that alpha-2-macroglobulin and hyaluronic acid might be useful markers of liver fibrosis (Tab. 2, Ref. 15). Text in PDF www.elis.sk.

KEY WORDS: chronic hepatitis C, fibromarkers, liver fibrosis, alpha-2-macroglobulin, hyaluronic acid.
\end{abstract}

\section{Introduction}

Liver fibrosis is the final common pathway of chronic liver diseases of various etiology (e.g. viral infections, alcohol abuse, autoimmune conditions or metabolic diseases). It is a complex dynamic process that includes an increase in extracellular matrix components, activation of cells producing matrix material, cytokine release and tissue remodelling. Progression to an advanced fibrosis can be rapid or slow, depending on disease state and the degree of activity of liver injury. The assessment of liver fibrosis is important for disease prognosis and for determination of the urgency of treatment as well as the response to therapy.

From the practical standpoint, it would be ideal to have a noninvasive fibromarker that can (a) stage the degree of liver fibrosis, (b) reflect the rate of matrix deposition or its removal, (c) monitor the impact of therapy. It should be (d) readily available, (e) reproducible and (f) inexpensive. Unfortunately, so far, none of the known and clinically used fibromarkers can meet all these requirements.

${ }^{1}$ Institute of Medical Chemistry, Biochemistry and Clinical Biochemistry, Medical School, Comenius University, Bratislava, Slovakia, and ${ }^{2} 3$ rd Department of Internal Medicine, Medical School, Comenius University and University Hospital, Bratislava, Slovakia

Address for correspondence: $\mathrm{L}$. Turecky, MD, $\mathrm{PhD}$, Institute of Medical Chemistry, Biochemistry and Clinical Biochemistry, Medical School, Comenius University, Sasinkova 2, SK-81108 Bratislava, Slovakia.

Acknowledgement: This study was financially supported by the VEGA grant No. 1/0808/15.
Despite of its invasive character, liver biopsy remains to be the "gold standard" in the diagnosis and staging of liver fibrosis. For both, the physician and the patient, the decision to proceed with liver biopsy is not trivial. Significant complications occur in $1-5 \%$ of patients with a reported mortality rate of between 1 : 1000 and $1: 10000$ (1). Due to the limitations of liver biopsy mentioned above, much effort has been invested to find a reliable serum liver fibromarker that would be a useful adjunct to clinical assessment and would reduce the need of repeated liver biopsy. Several biochemical candidates have been proposed as markers of fibrosis, either isolated or combined together in a scoring systems, such as PGA index, Fibro Test, Fibrometer test, Hepa Score or ELF score (2).

Liver fibrosis is a complex process involving production and deposition of different components that constitute the extracellular matrix (ECM). To these components belong proteins such as: collagens, noncollagenous glycoproteins (fibronectin, laminin, undulin, vitronectin, tenascin, osteonectin, elastin), proteoglycans and polysaccharide (hyaluronan)(3). Due to an increased production of ECM components in liver fibrosis, also the interest in determination of substances related to ECM as serum fibromarkers increases. One of the most useful fibromarkers is hyaluronan (hyaluronic acid, HA) (4-6). Hyaluronic acid is a chief component of ECM and plays an important structural role in the formation of ECM. Hyaluronic acid is a linear, anionic, nonsulfated glycosaminoglycan with unique characteristics. HA molecules are polymers of disaccharides (up to 25000 disaccharide repeat units in length) 
Tab. 1. Standard laboratory tests in patients with chronic hepatitis $\mathrm{C}$ and healthy controls.

\begin{tabular}{lccc}
\hline & ALT $\left(\mu \mathrm{kat} .1^{-1}\right)$ & AST $\left(\mu \mathrm{kat} .1^{-1}\right)$ & Cholinesterase $\left(\mu \mathrm{kat} .1^{-1}\right)$ \\
\hline Healthy controls & $0.32 \pm 0.07$ & $0.35 \pm 0.05$ & $66.02 \pm 13.33$ \\
Fibrosis grade 0 & $1.11 \pm 0.42$ & $0.62 \pm 0.25$ & $77.16 \pm 15.83$ \\
Fibrosis grade F1 & $1.22 \pm 0.53$ & $0.70 \pm 0.33$ & $74.52 \pm 13.92$ \\
Fibrosis grade F2 & $1.42 \pm 0.55$ & $0.78 \pm 0.32$ & $62.01 \pm 13.25$ \\
Fibrosis grade F3 & $0.78 \pm 0.48$ & $1.15 \pm 0.53$ & $37.8 \pm 4.2$ \\
Fibrosis grade F4 & $1.05 \pm 0.47$ & $1.18 \pm 0.48$ & $39.53 \pm 12.58$ \\
\hline
\end{tabular}

Results are given as the mean \pm SD (standard deviation)

composed of D-glucuronic acid and N-acetyl-D-glucosamine, linked via alternating $\beta(1 \rightarrow 4)$ and $\beta(1 \rightarrow 3)$ glycosidic bonds $(7)$.

Another potential serum fibromarker is alpha-2-macroglobulin (AMG). AMG is a large plasma protein with molecular weight approximately $720 \mathrm{kDa}$. It is a versatile protease inhibitor with a different mechanism than serpins. AMG has four identical subunits with molecular weight of $180 \mathrm{kDa}$, occurring as a pair of dimers. The dimers are disulfide-linked, whereas the pairs are held together by noncovalent bonds $(8,9)$. The dimer is the active unit, one molecule of AMG binds up to two protease molecules. AMG is synthesized primarily by hepatocytes and has a half-life of several days. After binding of the protease molecule, AMG-protease complex is cleared from circulation primarily by receptors on hepatocytes (10). The half-life of complexes is only a few minutes. The major function of AMG is the inhibition of proteases. AMG also binds to a variety of other proteins. AMG has been measured as one component of some multiparameter indices of hepatic fibrosis (11).

The aim of our study was to investigate the levels of alpha2-macroglobulin, potential fibromarker, in correlation to histological staging and to another potential serum fibromarker-hyaluronic acid - in patients with chronic hepatitis $\mathrm{C}$.

\section{Patients and methods}

\section{Study population}

Population groups under study consisted of 51 healthy volunteers - blood donors and 54 patients with chronic hepatitis C ( 37 men and 14 women, mean age 37.6 years). All patients had chronic hepatitis $\mathrm{C}$ virus (HCV) infection as confirmed by HCVRNA polymerase chain reaction analysis in serum. Coexisting liver diseases attributable to alcohol, hepatitis B, autoimmune hepatitis, primary biliary cirrhosis or haemochromatosis were reasonably excluded by history and standard clinical, laboratory, imaging and histologic studies. Human immunodeficiency virus coinfection and post-transplant patients were also excluded. Five $\mathrm{ml}$ of peripheral blood was taken from each patient in fasting state, one hour before performing liver biopsy. Liver biopsies were performed as part of routine clinical care in the staging and grading of liver disease. All patients and controls gave an informed consent for use of data and serum for research purposes. Local Ethics Committee approved the study.

\section{Liver biopsy}

Liver biopsies were obtained under an ultrasound guidance and were deemed adequate on the base of either specimen size or the
Tab. 2. Levels of alpha-2-macroglobulin and hyaluronic acid in patients with chronic hepatitis $\mathrm{C}$ and healthy controls.

\begin{tabular}{lcc}
\hline & $\begin{array}{c}\text { Alpha-2-macroglobulin } \\
\left(\mathrm{mg} .1^{-1}\right)\end{array}$ & $\begin{array}{c}\text { Hyaluronic acid } \\
\left(\mu \mathrm{gg} .1^{-1}\right)\end{array}$ \\
\hline Healthy controls & $1308 \pm 220$ & $15.52 \pm 9.37$ \\
Fibrosis grade F0 & $1375 \pm 210$ & $20.74 \pm 12.93$ \\
Fibrosis grade F1 & $1620 \pm 340$ & $25.75 \pm 11.5$ \\
Fibrosis grade F2 & $1820 \pm 310 * *$ & $34.53 \pm 18.0 *$ \\
Fibrosis grade F3 & $2810 \pm 565^{* * *}$ & $81.85 \pm 14.25^{* * *}$ \\
Fibrosis grade F4 & $3475 \pm 1115^{* * *}$ & $361.72 \pm 158.8 * * *$ \\
\hline Results are given as the mean $\pm \mathrm{SD}$, statistical significance $* \mathrm{p}<0.05 ; * * \mathrm{p}<0.01 ;$ \\
$* * * \mathrm{p}<0.001$
\end{tabular}

number of portal tracts. Biopsies were fixed, paraffin-embedded and stained with at least hematoxylin-eosin-safran and Masson's trichrome or picrosirius red for collagen. Biopsies were interpreted according to the scoring schema developed by METAVIR group (12). Fibrosis was scored on a 5-point scale: F0 - no fibrosis, F1 - portal fibrosis alone, F2 - portal fibrosis with rare septae, F3 portal fibrosis with many septae, F4 - cirrhosis.

\section{Laboratory methods}

Standard laboratory assessments including a complete blood cell count, routine biochemical parameters - ALT, AST, cholinesterase, albumin, bilirubin, were performed by a licensed clinical laboratory in fresh serum within 36 hours of collection. Other analyses were performed on sera stored frozen at $-80^{\circ} \mathrm{C}$. Alpha2-macroglobulin was determined immunochemically by electroimmunodiffusion according to Laurell (13), using monospecific antisera (Q-SwAHu/A2M, Sevapharma a.s., Praha, Czech republic). Hyaluronic acid was determined with an enzyme-linked binding protein assay that uses a capture molecule known as hyaluronic acid binding protein (Hyaluronic acid test kit, Corgenix, Inc., CO, USA).

\section{Statistical analysis}

Results are given as the mean $\pm \mathrm{SD}$ (standard deviation). Means were compared using the non-parametric Wilcoxon test. We have considered $p$ values of less than 0.05 to be statistically significant.

\section{Results}

The results of standard biochemical tests are presented in the Table 1. Albumin and cholinesterase, parameters reflecting the liver function, are in the groups F0 and F1 not significantly different from healthy controls. In the patients with fibrosis grade F2, these parameters are slightly decreased. Albumin level and 
cholinesterase activity were significantly decreased in the group F4 $(\mathrm{p}<0.001)$.

Both potential fibromarkers - hyaluronic acid and alpha2-macroglobulin - were increased in patients with HCV infection (Tab. 2). The level of alpha-2-macroglobulin in the group F0 was practically the same as in healthy controls. The level of alpha-2-macroglobulin was slightly increased in the group F1 compared to healthy controls, but this difference was statistically not significant. In the groups F2-F4, AMG levels were significantly higher than in the control group. The differences between F2 - F3 $\left(1820 \pm 310 \mathrm{mg} . \mathrm{l}^{-1}\right.$ vs $\left.2810 \pm 565 \mathrm{mg} \cdot \mathrm{l}^{-1}\right)$ and F3 $-\mathrm{F} 4(2810 \pm 565$ mg. $1^{-1}$ vs $\left.3475 \pm 1115 \mathrm{mg} .1^{-1}\right)$ were also statistically significant.

The results of hyaluronan determination showed that there was no difference between the group F0 and healthy controls (20.74 \pm $12.93 \mu \mathrm{g} .1^{-1}$ vs $\left.15.52 \pm 9.37 \mu \mathrm{g} .1^{-1}\right)$. The levels of hyaluronan were slightly increased in the group F1 and F2 compared to the control group $\left(25.75 \pm 11.5 \mu \mathrm{g} . \mathrm{l}^{-1}\right.$, resp. $34.53 \pm 18 \mu \mathrm{g} . \mathrm{l}^{-1}$ vs $15.52 \pm 9.37$ $\left.\mu \mathrm{g} . \mathrm{l}^{-1}\right)$, but the difference between the group F1 and F2 was not significant. The levels of HA in the group F3 $\left(81.85 \pm 14.25 \mu \mathrm{g} . \mathrm{l}^{-1}\right.$ vs $\left.15.52 \pm 9.37 \mu \mathrm{g} .1^{-1}\right)$ and the group F4 (361.72 $\pm 159.8 \mu \mathrm{g} .1^{-1}$ vs $\left.15.52 \pm 9.37 \mu \mathrm{g} .1^{-1}\right)$ were significantly increased in comparison with the control group. The levels of HA in the groups F3 and F4 were also significantly higher than in the group F2.

Regression analysis of the relationship of hyaluronic acid and alpha-2-macroglobulin levels showed a statistically significant correlation between these two parameters in sera of patients with chronic hepatitis $\mathrm{C}(\mathrm{r}=0.43, \mathrm{p}<0.001)$.

\section{Discussion}

Chronic hepatitis $\mathrm{C}$ is a progressive disease. Because the prognosis of patients with this disease depends mainly on the development of liver fibrosis, this process needs to be regularly evaluated. Despite the fact that histological examination is the „gold standard“ for assessing liver fibrosis, liver biopsy is potentially associated with severe complications and for ethical reasons cannot be repeated to monitor liver status. As a consequence of the difficulties linked to liver histological examination, serum fibromarkers are now used for monitoring of the liver fibrosis in patients with HCV infection. Developing an efficient and noninvasive method for liver fibrosis is important for prognosis and treatment of patients with chronic hepatitis type C. A noninvasive diagnosis of liver fibrosis could also enhance the development of antifibrotic therapies.

There are several types of non-invasive markers utilized for evaluation of severity of fibrosis in patients with chronic hepatopathy. Indirect markers utilize routine laboratory parameters such as: transaminases, albumin, prothrombin time or other parameters, which relate to the liver disease stage. Direct serum markers of fibrosis are laboratory parameters, which reflect the ECM turnover and can be used for staging of fibrosis, and also for monitoring the progression or regression of liver fibrosis. These markers include several glycoproteins, collagens, collagenases and their inhibitors. These have been studied individually as well as in combinations in different scoring systems.
In the present study, we have evaluated and compared alpha2-macroglobulin and hyaluronan serum levels as markers of liver fibrosis. Hyaluronan is the most studied direct serum marker. It is a glycosaminoglycan that is synthesized in extracellular space by hepatic stellate cells and is degraded by sinusoidal endothelial cells (14). The results of our study showed that the levels of HA in the group of patients with chronic hepatitis $\mathrm{C}$ were increased, when compared to the healthy controls. This increase correlates well with the severity of liver fibrosis. Patients with mild fibrosis (fibrosis grade $\leq$ F2) had only slightly increased levels of HA, but this increase was not significantly different from reference values. Significantly increased levels of HA were found in the patients with an extensive liver fibrosis (fibrosis grade F3 and F4). The results indicated that the hyaluronic acid level used as the fibromarker is a more reliable diagnostic tool in patients with more severe fibrosis than in patients with moderate form of fibrosis. The explanation of increased levels of HA in patients with liver fibrosis could be the activation of hepatic stellate cells, which is characteristic for liver fibrosis, and/or the endothelial dysfunction that occurs as fibrosis progresses. Our results of investigation of hyaluronic acid levels in patients with chronic HCV infection are in good agreement with results presented by Guechot et al (4). These authors presented cut off values for diagnosing extensive liver fibrosis in patients with chronic hepatitis $\mathrm{C}$ as $85 \mu \mathrm{g} .1^{-1}$. The mean value of HA in the group F3 in our patients is practically the same $-82 \mu \mathrm{g} \cdot 1^{-1}$.

The second investigated parameter, alpha-2-macroglobulin, was also increased in our group of patients with chronic hepatitis C. The changes of AMG levels were similar to changes of hyaluronic acid. In the group of patients with F0 fibrosis, there were no changes compared to the control group. In the patients with a slight grade of fibrosis (F1-F2), the levels of AMG were moderately increased, but they were still in the reference range. The significant increase in AMG levels was found only in patients with severe grade of fibrosis (F3-F4). One of the possible explanations of increased AMG levels in the patients with liver fibrosis is associated with the function of this protein. AMG is one of the major antiproteinases, present in plasma of vertebrates. Another important property of AMG is its ability to bind a variety of other proteins. AMG binds several cytokines and growth factors. The biological activity of some cytokines is inhibited when bound to AMG. During liver fibrosis, TGF $\beta$ (transforming growth factor beta) stimulates the production of matrix. It has been shown earlier that AMG inhibits the activity of TGF $\beta$. Tiggelman et al (15) observed that TGF $\beta$-induced collagen formation by human liver myofibroblasts is reduced in vitro by AMG. It is possible, that AMG may have an anti-fibrogenic effect on TGF $\beta$-induced activation of stellate cells and the increase of AMG levels in patients with liver fibrosis is a compensation mechanism decreasing the production of extracellular matrix. Another mechanism for the increase of AMG levels in patients with liver fibrosis is a decreased clearance of AMG-protein complexes. These complexes are cleared from circulation primarily by receptors on hepatocytes (10). Damage of hepatocytes in chronic hepatopathies and disorders of the structure of hepatic sinusoids, as a consequence 
of accumulation of extracellular matrix in perisinusoidal space, may lead to a decreased uptake of alpha-2-macroglobulin-protein complexes by hepatocytes leading to an increase of plasma alpha2-macroglobulin levels.

The similarity of changes of alpha-2-macroglobulin and hyaluronic acid levels was confirmed also by the regression analysis, which showed the statistically significant correlation between these two parameters $(\mathrm{p}<0.001)$

According to the results of our study, we can conclude that the determination of alpha-2-macroglobulin and hyaluronic acid plasma levels may be a useful diagnostic tool in the verification of liver fibrosis and in the monitoring of the activity of hepatic fibrogenesis. Our results showed that these parameters are more reliable in patients with more severe grade of fibrosis. In regard to an interindividual variability of these parameters, their utilization is more favorable for monitoring the fibrogenic activity and effects of therapy than for the determination of the grade of liver fibrosis.

\section{References}

1. Afdhal NH, Nunes D. Evaluation of liver fibrosis. A concise review. Amer J Gastroenterol 2004; 99 (6): 1160-1174.

2. Sherwood RA, Bomford A. Assessment of hepatic function and investigation of jaundice. 231-249. In: Marshall WJ, Lapsley M, Day AP, Ayling RM (Eds). Clinical Biochemistrry. London: Churchill Livingstone, Elsevier, 2014.

3. Gressner AM. Activation of proteoglycan synthesis in injured livera brief review of molecular and cellular aspects. Eur J Clin Chem Biochem 1994; 32 (4): 225-237.

4. Guéchot J, Laudat A, Loria A, Serfaty L, Poupon R, Giboudeau J. Diagnostic accuracy of hyaluronan and type III procollagen amino-terminal peptide serum assays as markers of liver fibrosis in chronic hepatitis C evaluated by ROC curve analysis. Clin Chem 1996; 42 (4): 558-563.
5. Orasan OH, Ciulei G, Cozma A, Sava M, Dumitrascu DL. Hyaluronic acid as a biomarker of fibrosis in chronic liver diseases of different etiologies. Clujul Medical 2016; 89 (1): 24-31.

6. Gudowska M, Cylwik O, Chrostek L. The role of serum hyaluronic acid determination in the diagnosis of liver fibrosis. Acta Biochim Polon 2017; 64: Epub: No 2016_1443.

7. Kaux JF, Samson A, Crielaard JM. Hyaluronic acid and tendon lesions. Muscles Ligaments Tendons J 2016; 5 (4): 264-269.

8. Sheikh AM, Chauhan V, Tsiouris JA et al. Elevated levels of serum alpha2 macroglobulin in wild black bears during hibernation. Biochimie 2003; 85: 1027-1032.

9. Wyatt AR, Zammit NW, Wilson MR. Acute phase proteins are major clients for the chaperone action of alpha-2-macroglobulin in human plasma. Cell Stress Chaperones 2013; 18 (2): 161-170.

10. Bond JE, Cianciolo GJ, Pizzo SV. Incorporation of low molecular weight molecules into alpha-2-macroglobulin by nucleophilic exchange. Biochem Biophys Res Commun 2007; 357 (2): 433-438.

11. Gressner OA, Weiskirchen R, Gressner AM. Biomarkers of liver fibrosis: clinical translation of molecular pathogenesis or based on liver dependent multifunction tests. Clin Chim Acta 2007; 381 (2): 107-113.

12. Bedossa P, Poynard T. An algorithm for the grading of activity in chronic hepatitis C: the METAVIR Cooperative Study Group. Hepatology 1996; 24 (2): 289-293.

13. Laurell CB. Quantitative estimation of proteins by electrophoresis in agarose gel containing antibodies. Anal Biochem 1966; 15 (1): 45-52.

14. Shiha G. Serum hyaluronic acid: a promising marker of hepatic fibrosis in chronic hepatitis B. Saudi J Gastroenterol 2008; 14 (4): 161-162.

15. Tiggelman AM, Linthorst C, Boers W, Brand HS, Chamuleau RA. Transforming ghrowth factor- $\beta$-induced collagen synthesis by human liver myofibroblasts is inhibited by alpha-2 macroglobulin. J Hepatol 1997; 26 (6): 1220-1228.

Received August 1, 2017. Accepted September 4, 2017. 